\title{
Reconstruction of Past Failure Times for Left Type-II Censored Data from Weibull Model
}

\author{
A. Asgharzadeh ${ }^{\dagger, *}$, Z. Mirzazadeh Ganji ${ }^{\dagger}$, R. Valiollahi ${ }^{\ddagger}$ and J. Ahmadi ${ }^{\star}$ \\ † University of Mazandaran \\ $\ddagger$ Semnan University \\ * Ferdowsi University of Mashhad,
}

Received: 9/18/2016 Approved: 6/7/2017

\begin{abstract}
This paper deals with the problem of reconstructing missing data in a left type-II censoring scheme, where the underlying distribution is the Weibull distribution. Frequentist and Bayesian approaches are adopted in order to provide some point reconstructors for the past failure times. The problem of determining reconstruction intervals for the past failure times is also considered. The investigation includes an example of application to real data and various comparisons based on Monte Carlo simulations.
\end{abstract}

Keywords. Left censoring; maximum likelihood reconstructor; best unbiased and conditional median reconstructors; reconstruction interval.

MSC 2010: 62G30; 62N01.

\section{Introduction}

Censored sampling arises in a life testing experiment whenever the experimenter does not observe (either intentionally or unintentionally) the failure times of all units placed on a life test. Suppose $n$ components are put to

Corresponding author 
test simultaneously at time 0 , and the failure times of these components are recorded. But sometimes, the failure times are not observed continuously. This can happen because the experimenter might not always be in a position to observe the lifetimes of all the items put on test due to time limitations or other restrictions. Let us suppose that out of $n$ items put on life test experiment, only the largest $n-r$ life times have been observed and the failure times for the first $r$ components remain unobserved or missing. In the literature of reliability and survival analysis, this type of censoring is known as a left type-II censoring scheme. There are many applications of left type-II censored data in the analysis of survival data. For example, in medical studies, we may know the date of a medical exam that revealed a disease such as cancer, but we don't know when the patient has been infected. In scientific experiments, we may not be able to measure some quantity because it is below the threshold of detection (e.g. chemical concentration). In behavioral sciences, we may know that teenagers are subject to alcohol, but we don't know the exact time at which teenagers begin to drink alcohol. However, as mentioned by Mitra and Kundu (2008), discovery of a condition only tells us that the onset of sickness fell in the period since the previous examination and nothing about the exact date of the attack. Thus the time elapsed since onset has been left censored. For more details and some other applications of left type-II censoring scheme, see Balakrishnan (1989); Balakrishnan and Varadan (1991); Scallan (1999); Bagger (2005); Mitra and Kundu (2008); Bhaumik et al. (2009) and Noor and Aslam (2013).

It is obvious that in left type-II censored plan, the life test experiment has been conducted on a random sample of $n$ items. We have missed the contained information in the first failure times, so it is worthwhile, if one can reconstruct the missing failure times. Reconstruction of the past failure times in the left censored setup is an interesting topic which is used in actuarial, medical and engineering sciences. In the recent years, several authors have considered reconstruction problems involving order statistics and record values. Klimczak and Rychlik (2005) obtained upper bounds for the expectations of increments of order statistics and record values under the condition that the values of future order statistics and records are available. Balakrishnan et al. (2009) have addressed the problem of reconstructing past records from the known values of future records when the underlying distributions were exponential and Pareto distributions. Razmkhah et al. (2010) have derived point and interval reconstructors for the missing order statistics from two parameter exponential distribution. Asgharzadeh et al.

() 2017, SRTC Iran 
(2012) discussed reconstructors of times to failure of units censored in a leftcensored sample from the proportional reversed hazard rate models. Khatib et al. (2013) have considered the Bayesian reconstruction of the missing failure times in exponential distribution. Asgharzadeh et al. (2014) studied the estimation and reconstruction problems for the two-parameter Pareto distribution based on the left censored data. El-Adll and Aly (2016) obtained reconstructors for past fractional upper (lower) records from exponential and Frećhet distributions.

Suppose that out of a total of $n$ observations, we only observe the largest $n-r$ life times $X_{r+1}<X_{r+2}<\cdots<X_{n}$ and $r$ observations are censored on the left. Our main aim is to reconstruct the past failure times $X_{1}<X_{2}<$ $\cdots<X_{r}$ based on the $n-r$ observed order statistics $\mathbf{X}=\left(X_{r+1}, \ldots, X_{n}\right)$. In what follows, we intend to study this problem for Weibull distribution and present several reconstructors of $Y=X_{s},(1 \leqslant s \leqslant r)$ via non-Bayesian and Bayesian approaches. The Weibull model, having exponential and Rayleigh as special sub-models, appears very frequent in practical problems when we observe data representing failure times. It is used for modeling lifetime data and analysis of survival data.

The rest of the paper is organized as follows. Section 2 contains some preliminaries. In Section 3, the maximum likelihood reconstructor is derived for the past failure times. Best unbiased and conditional median reconstructors are provided in Section 4. The Bayesian reconstructor is derived in Section 5 and it is observed that the Bayesian reconstructor cannot be obtained in a closed form and a simple sampling technique is used to find an approximate for it. In Section 6, some reconstruction intervals (RI's) for the past failure times based on Bayesian and non-Bayesian approaches are given. In Section 7 , a real data set analysis is presented for illustrative purposes. Finally in Section 8, numerical comparisons are made between proposed reconstructors using Monte Carlo simulations.

\section{Some Preliminaries}

Let $\mathbf{X}=\left(X_{r+1}, X_{r+2}, \ldots, X_{n}\right)$ be the available left type-II censored sample from a random sample of size $n$ from a life-time distribution with cumulative distribution function (cdf) $F(x ; \eta)$ and probability density function (pdf) $f(x ; \eta)$, where $\eta$ represents the vector of unknown parameters. Then, the 
joint pdf of $\mathbf{X}=\left(X_{r+1}, X_{r+2}, \ldots, X_{n}\right)$ is given by

$$
f(\mathbf{x} ; \eta)=\frac{n !}{r !}\left[F\left(x_{r+1} ; \eta\right)\right]^{r} \prod_{i=r+1}^{n} f\left(x_{i} ; \eta\right), \quad x_{r+1}<x_{r+2}<\cdots<x_{n},
$$

where $\mathbf{x}=\left(x_{r+1}, x_{r+2}, \ldots, x_{n}\right)$ is the vector of observations. Our objective is to reconstruct the $s$ th past failure time $Y=X_{s}(1 \leqslant s \leqslant r)$ based on the observed censored sample $\mathbf{X}=\left(X_{r+1}, \ldots, X_{n}\right)$. Due to Markovian property of order statistics, the conditional distribution of $Y=X_{s}$, given $\mathbf{x}=$ $\left(x_{r+1}, \ldots, x_{n}\right)$ is equal to the conditional distribution of $Y$ given $X_{r+1}=x_{r+1}$ which is given by

$f\left(y \mid x_{r+1} ; \eta\right)=s\left(\begin{array}{c}r \\ s\end{array}\right) f(y ; \eta)[F(y ; \eta)]^{s-1}\left[F\left(x_{r+1 ; \eta}\right)-F(y ; \eta)\right]^{r-s}\left[F\left(x_{r+1 ; \eta}\right)\right]^{-r}$

for $y \leqslant x_{r+1}$. The expression in (2) can be rewritten as

$$
f\left(y \mid x_{r+1} ; \eta\right)=s\left(\begin{array}{c}
r \\
s
\end{array}\right)\left[\frac{F(y ; \eta)}{F\left(x_{r+1 ; \eta}\right)}\right]^{s-1}\left[1-\frac{F(y ; \eta)}{F\left(x_{r+1 ; \eta}\right)}\right]^{r-s} \frac{f(y ; \eta)}{F\left(x_{r+1 ; \eta}\right)} .
$$

Now, take $U=\left(\frac{F(Y ; \eta)}{F\left(x_{r+1 ; \eta}\right)}\right)$, then the conditional pdf of $U$ given $X_{r+1}=x_{r+1}$ is

$$
g\left(y \mid x_{r+1} ; \eta\right)=\frac{\Gamma(r+1)}{\Gamma(s) \Gamma(r-s+1)} u^{s-1}(1-u)^{r-s}, \quad 0<u<1,
$$

i.e. $U \mid X_{r+1}=x_{r+1}$ has beta distribution with parameters $s$ and $r-s+1$.

In what follows, we assume that $X_{r+1}, X_{r+2}, \ldots, X_{n}$ are the available left type-II censored data from a random sample of size $n$ from a two-parameter Weibull distribution (denoted by $W(\lambda, \theta)$ ) with cdf

$$
F(x ; \lambda, \theta)=1-\exp \left(-x^{\lambda} / \theta\right), \quad x \geqslant 0,
$$

where $\lambda$ and $\theta$ are positive real values. It is known that the Weibull distribution is one of the most widely used distributions in reliability and survival analysis which includes the exponential and Rayleigh distributions as special case. Because of the shape and scale parameters, it has been used very effectively for analyzing lifetime data, particularly when the data are censored, which is very common in most life testing experiments. It also has increasing and decreasing failure rates depending on the shape parameter. A detailed 
discussion on Weibull model can be found in Johnson et al. (1994).

From equations (1) and (5) the joint pdf of $\mathbf{X}=\left(X_{r+1}, X_{r+2}, \ldots, X_{n}\right)$ is given by

$$
\begin{aligned}
f(\mathbf{x} ; \lambda, \theta)= & \frac{n !}{r !}(\lambda / \theta)^{n-r}\left[1-\exp \left(-x_{r+1}^{\lambda} / \theta\right)\right]^{r} \exp \left[-\sum_{i=r+1}^{n} x_{i}^{\lambda} / \theta\right] \prod_{i=r+1}^{n} x_{i}^{\lambda-1} \\
& 0 \leqslant x_{r+1}<\cdots<x_{n} .
\end{aligned}
$$

Then, the log-likelihood function is given by

$$
\begin{aligned}
\ln L(\lambda, \theta) \propto & (n-r) \ln \left(\frac{\lambda}{\theta}\right)+r \ln \left[1-\exp \left(-\frac{x_{r+1}^{\lambda}}{\theta}\right)\right]+(\lambda-1) \sum_{i=r+1}^{n} \ln x_{i} \\
& -\sum_{i=r+1}^{n} x_{i}^{\lambda} / \theta
\end{aligned}
$$

From (7), the maximum likelihood estimators (MLEs) of $\lambda$ and $\theta$ can be obtained by solving the following likelihood equations:

$$
\begin{gathered}
\frac{\partial \ln L(\lambda, \theta)}{\partial \lambda}=\frac{n-r}{\lambda}+\frac{r x_{r+1}^{\lambda} \ln \left(x_{r+1}\right)}{\theta} \frac{\exp \left(-x_{r+1}^{\lambda} / \theta\right)}{1-\exp \left(-x_{r+1}^{\lambda} / \theta\right)} \\
-\frac{1}{\theta} \sum_{i=r+1}^{n} x_{i}^{\lambda} \ln x_{i}+\sum_{i=r+1}^{n} \ln x_{i}=0 \\
\frac{\partial \ln L(\lambda, \theta)}{\partial \theta}=-\frac{(n-r)}{\theta}-\frac{r}{\theta^{2}} x_{r+1}^{\lambda} \frac{\exp \left(-x_{r+1}^{\lambda} / \theta\right)}{1-\exp \left(-x_{r+1}^{\lambda} / \theta\right)}+\frac{1}{\theta^{2}} \sum_{i=r+1}^{n} x_{i}^{\lambda}=0 .
\end{gathered}
$$

It is observed that numerical computations are needed to obtain $\hat{\lambda}$ and $\widehat{\theta}$. Also, for the Weibull distribution with cdf in (5), the identity (3) reduces to

$$
\begin{aligned}
f\left(y \mid x_{r+1} ; \lambda, \theta\right) & =s\left(\begin{array}{l}
r \\
s
\end{array}\right)\left(\frac{\lambda}{\theta}\right) y^{\lambda-1} \exp \left(-\left(y^{\lambda} / \theta\right)\right)\left[1-\exp \left(-y^{\lambda} / \theta\right)\right]^{s-1} \\
& \times\left[\exp \left(-y^{\lambda} / \theta\right)-\exp \left(-x_{r+1}^{\lambda} / \theta\right)\right]^{r-s} \\
& \times\left[1-\exp \left(-x_{r+1}^{\lambda} / \theta\right)\right]^{-r}, \quad y \leqslant x_{r+1} .
\end{aligned}
$$

In the next sections, we use (10) to construct some reconstructors. 


\section{Maximum Likelihood Reconstructor}

Here, we use the likelihood approach to obtain maximum likelihood reconstructor (MLR). The likelihood approach was suggested by Kaminsky and Rhodin (1985) for predicting future observations. See also Raqab and Nagaraja (1995) and Raqab et al. (2010). We use this approach for reconstructing the past failure times. Let $\mathbf{X}=\left(X_{r+1}, \ldots, X_{n}\right)$ and $Y=X_{s}(1 \leqslant s \leqslant r)$ have the joint pdf $f(x, y ; \eta)$ indexed by the parameter $\eta \in R^{k}$. Here, our aim is reconstructing the past random variable $Y$, based on observed $\mathbf{x}=\left(x_{r+1}, \ldots, x_{n}\right)$. The reconstructive likelihood function (RLF) of $Y$ and $\eta$ is given by

$$
L(y, \eta)=f(\mathbf{x}, y ; \eta)=f(y \mid \mathbf{x} ; \eta) f(\mathbf{x} ; \eta) .
$$

Suppose $\hat{Y}=u(\mathbf{X})$ and $\hat{\eta}=v(\mathbf{X})$ are statistics for which

$$
L(u(\mathbf{x}), v(\mathbf{x}))=\sup _{(y, \eta)} L(y, \eta),
$$

then $u(\mathbf{X})$ and $v(\mathbf{X})$ are, respectively, called the MLR of $Y$ and the reconstructive maximum likelihood estimator (RMLE) of $\eta$.

For the Weibull distribution, using (6) and (10), we derive the RLF of $Y$ and $(\lambda, \theta)$ for $y \leqslant x_{r+1}$ as

$$
\begin{aligned}
L(y, \lambda, \theta)= & c\left(\frac{\lambda}{\theta}\right)^{n-r+1} \exp \left(-\frac{1}{\theta}\left[y^{\lambda}+\sum_{i=r+1}^{n} x_{i}^{\lambda}\right]\right) y^{\lambda-1} \prod_{i=r+1}^{n} x_{i}^{\lambda-1} \\
& \times\left[1-\exp \left(-y^{\lambda} / \theta\right)\right]^{s-1}\left[\exp \left(-y^{\lambda} / \theta\right)-\exp \left(-x_{r+1}^{\lambda} / \theta\right)\right]^{r-s}
\end{aligned}
$$

where $c=\frac{n !}{(s-1) !(r-s) !}$. Apart from a constant term, the reconstructive loglikelihood function of $Y$ and $(\lambda, \theta)$ is

$$
\begin{aligned}
\ln L(y, \lambda, \theta) \propto & (n-r+1) \ln \left(\frac{\lambda}{\theta}\right)-\frac{1}{\theta}\left[y^{\lambda}+\sum_{i=r+1}^{n} x_{i}^{\lambda}\right] \\
& +(\lambda-1)\left[\ln (y)+\sum_{i=r+1}^{n} \ln \left(x_{i}\right)\right]+(s-1) \ln \left[1-\exp \left(-y^{\lambda} / \theta\right)\right] \\
& +(r-s) \ln \left[\exp \left(-y^{\lambda} / \theta\right)-\exp \left(-x_{r+1}^{\lambda} / \theta\right)\right]
\end{aligned}
$$


From (12), the reconstructive likelihood equations (RLEs) for $y, \lambda$ and $\theta$ are given, respectively, by

$$
\begin{aligned}
\frac{\partial \ln L(y, \lambda, \theta)}{\partial y}= & -\frac{\lambda}{\theta} y^{\lambda-1}+\frac{\lambda-1}{y}+(s-1) \frac{\lambda y^{\lambda-1}}{\theta} \times \frac{\exp \left(-y^{\lambda} / \theta\right)}{1-\exp \left(-y^{\lambda} / \theta\right)} \\
& -(r-s) \frac{\lambda y^{\lambda-1}}{\theta} \times \frac{\exp \left(-y^{\lambda} / \theta\right)}{\exp \left(-y^{\lambda} / \theta\right)-\exp \left(-x_{r+1}^{\lambda} / \theta\right)} \\
= & 0
\end{aligned}
$$

$$
\begin{aligned}
\frac{\partial \ln L(y, \lambda, \theta)}{\partial \lambda} & =\frac{(n-r+1)}{\lambda}-\frac{1}{\theta}\left[y^{\lambda} \ln y+\sum_{i=r+1}^{n} x_{i}^{\lambda} \ln \left(x_{i}\right)\right] \\
& +\left[\ln (y)+\sum_{i=r+1}^{n} \ln \left(x_{i}\right)\right]+(s-1) \frac{y^{\lambda} \ln (y)}{\theta} \times \frac{\exp \left(-y^{\lambda} / \theta\right)}{1-\exp \left(-y^{\lambda} / \theta\right)} \\
& +\frac{r-s}{\theta} \frac{x_{r+1}^{\lambda} \ln \left(x_{r+1)} \exp \left(-x_{r+1}^{\lambda} / \theta\right)-y^{\lambda} \ln (y) \exp \left(-y^{\lambda} / \theta\right)\right.}{\exp \left(-y^{\lambda} / \theta\right)-\exp \left(-x_{r+1}^{\lambda} / \theta\right)} \\
& =0
\end{aligned}
$$

and

$$
\begin{aligned}
\frac{\partial \ln L(y, \lambda, \theta)}{\partial \theta}= & -\frac{(n-r+1)}{\theta}+\frac{1}{\theta^{2}}\left[y^{\lambda}+\sum_{i=r+1}^{n} x_{i}^{\lambda}\right] \\
- & (s-1) \frac{y^{\lambda}}{\theta^{2}} \frac{\left(\exp \left(-y^{\lambda} / \theta\right)\right.}{1-\left(\exp \left(-y^{\lambda} / \theta\right)\right)} \\
& +\frac{(r-s)}{\theta^{2}} \times \frac{y^{\lambda} \exp \left(-y^{\lambda} / \theta\right)-x_{r+1}^{\lambda} \exp \left(-x_{r+1}^{\lambda} / \theta\right)}{\exp \left(-y^{\lambda} / \theta\right)-\exp \left(-x_{r+1}^{\lambda} / \theta\right)} \\
= & 0 .
\end{aligned}
$$


By combining the three equations, we obtain the MLR of $Y$ as

$$
\hat{Y}_{M L R}=\left(x_{r+1}^{\widetilde{\lambda}}-\widetilde{\theta} \ln \left[\frac{(r-s) x_{r+1}^{\tilde{\lambda}}}{\widetilde{\theta}\left(\frac{\tilde{\lambda}-1}{\tilde{\lambda}}-(n-r+1)\right)+\sum_{i=r+1}^{n} x_{i}^{\tilde{\lambda}}}+1\right]\right)^{1 / \widetilde{\lambda}},
$$

where $(\tilde{\lambda}, \tilde{\theta})$ is RMLE of $(\lambda, \theta)$, that can be obtained numerically from two equations (14) and (15), if we replace $y$ in terms of $\tilde{\lambda}$ and $\tilde{\theta}$ from (16).

\section{Best Unbiased and Conditional Median Recon- structors}

The conditional distribution of $Y$ given $X_{r+1}=x_{r+1}$ is used to obtain the best unbiased and conditional median reconstructors. Following Asgharzadeh et al. (2012), the mean of $Y$ given $X_{r+1}=x_{r+1}$ is the best unbiased reconstructor (BUR) of $Y$. Therefore, the BUR of $Y$ is

$$
\widehat{Y}_{B U R}=\int_{0}^{x_{r+1}} y f\left(y \mid x_{r+1}, \lambda, \theta\right) d y .
$$

Substituting (10) into (17), and setting $u=\left(\frac{1-\exp \left(-y^{\lambda} / \theta\right)}{1-\exp \left(-x_{r+1}^{\lambda} / \theta\right)}\right)$, after some simplifications, the the BUR of $Y$ can be presented as

$$
\widehat{Y}_{B U R}=\int_{0}^{1}\left(-\theta \ln \left[1-u\left(1-\exp \left(-x_{r+1}^{\lambda} / \theta\right)\right)\right]\right)^{1 / \lambda} \frac{u^{s-1}(1-u)^{r-s}}{\operatorname{Beta}(s, r-s+1)} d u .
$$

If the parameters $\lambda$ and $\theta$ are unknown, they have to be estimated. Thus one would replace $\lambda$ and $\theta$ by their corresponding MLEs.

Also, the median of $Y$ given $\mathbf{X}$ is known as the conditional median reconstructor (CMR). So, a reconstructor $\hat{Y}$ of $Y$ is the CMR of $Y$ if

$$
P_{\lambda, \theta}(Y \leqslant \hat{Y} \mid \mathbf{X}=\mathbf{x})=P_{\lambda, \theta}(Y \geqslant \hat{Y} \mid \mathbf{X}=\mathbf{x}) .
$$

By (4) for the Weibull distribution, it follows that

$$
U=\left(\frac{1-\exp \left(-Y^{\lambda} / \theta\right)}{1-\exp \left(-x_{r+1}^{\lambda} / \theta\right)}\right) \mid X_{r+1}=x_{r+1} \sim \operatorname{Beta}(s, r-s+1) .
$$


Now, since the probability $P_{\lambda, \theta}\left(Y \geqslant \hat{Y} \mid X_{r+1}=x_{r+1}\right)$ is equivalent to

$$
P_{\lambda, \theta}\left(\frac{1-\exp \left(-Y^{\lambda} / \theta\right)}{1-\exp \left(-x_{r+1}^{\lambda} / \theta\right)} \geqslant \frac{1-\exp \left(-\hat{y}^{\lambda} / \theta\right)}{1-\exp \left(-x_{r+1}^{\lambda} / \theta\right)} \mid X_{r+1}=x_{r+1}\right)
$$

the CMR of $Y$ is obtained by solving the following equation for $\hat{Y}$ :

$$
\frac{1-\exp \left(-\hat{Y}^{\lambda} / \theta\right)}{1-\exp \left(-x_{r+1}^{\lambda} / \theta\right)}=\operatorname{Med}(U)
$$

where $\operatorname{Med}(U)$ is the median of the beta random variable $U \sim \operatorname{Beta}(s, r-$ $s+1$ ). From (21), the CMR of $Y$ is

$$
\hat{Y}_{C M R}=\left[-\theta \ln \left[1-\operatorname{Med}(U)\left(1-\exp \left(-x_{r+1}^{\lambda} / \theta\right)\right)\right]\right]^{1 / \lambda} .
$$

If $\lambda$ and $\theta$ are unknown, we can substitute $\lambda$ and $\theta$ by their corresponding MLEs.

For the special case $s=r$, we have $\operatorname{Med}(U)=1 / \sqrt[r]{2}$, and hence we obtain

$$
\hat{Y}_{C M R}=\left[-\theta \ln \left[1-(1 / 2)^{1 / r}\left(1-\exp \left(-x_{r+1}^{\lambda} / \theta\right)\right)\right]\right]^{1 / \lambda} .
$$

\section{Bayesian Reconstructor}

In this section, our interest is to reconstruct the $s$ th past failure time $Y=$ $X_{s}(1 \leqslant s \leqslant r)$ based on the observed censored sample $\mathbf{X}=\left(X_{r+1}, \ldots, X_{n}\right)$ from a Bayesian approach. Following Aitchison and Dunsmore (1975), the Bayes reconstructive density of $Y=X_{s}$ given $\mathbf{x}$ is

$$
f_{s}^{*}(y \mid \mathbf{x})=\int_{0}^{\infty} \int_{0}^{\infty} f(y \mid \mathbf{x}, \theta, \lambda) \pi(\theta, \lambda \mid \mathbf{x}) d \theta d \lambda,
$$

where $f(y \mid \mathbf{x}, \theta, \lambda)$ is the conditional density of $Y=X_{s}$ given $\mathbf{x}=\left(x_{r+1}, \ldots, x_{n}\right)$ and $\pi(\theta, \lambda \mid \mathbf{x})$ is the joint posterior density of $\theta$ and $\lambda$. We take independent priors for $\lambda$ and $\theta$ which are gamma, $\Gamma\left(a_{1}, b_{1}\right)$, and inverse gamma, $\operatorname{I}\left(a_{2}, b_{2}\right)$, with density functions as

$$
\pi(\lambda)=\frac{\lambda^{a_{1}-1} b_{1}^{a_{1}}}{\Gamma\left(a_{1}\right)} e^{-b_{1} \lambda} \quad \text { and } \quad \pi(\theta)=\frac{\theta^{-\left(a_{2}+1\right)} b_{2}^{a_{2}}}{\Gamma\left(a_{2}\right)} e^{-\frac{b_{2}}{\theta}}
$$


respectively, where $a_{1}, b_{1}, a_{2}$ and $b_{2}$ are all positive. Then, the joint posterior density function of $\theta$ and $\lambda$ given the data can be written as

$$
\pi(\theta, \lambda \mid \mathbf{x}) \propto g_{1}(\theta \mid \lambda, \mathbf{x}) g_{2}(\lambda \mid \mathbf{x}) h(\theta, \lambda ; \mathbf{x}),
$$

see for example Kundu $(2007,2008)$. Here $g_{1}(\theta \mid \lambda, \mathbf{x})$ is an inverse gamma density function with the shape and scale parameters as $n-r+a_{2}$ and $\sum_{i=r+1}^{n} x_{i}^{\lambda}+b_{2}$ respectively,

$$
h(\theta, \lambda ; \mathbf{x})=\left(1-\exp \left(-x_{r+1}^{\lambda} / \theta\right)\right)^{r}
$$

and

$$
g_{2}(\lambda \mid \mathbf{x}) \propto \frac{\lambda^{n-r+a_{1}-1} e^{-b_{1} \lambda}}{\left(\sum_{i=r+1}^{n} x_{i}^{\lambda}+b_{2}\right)^{n-r+a_{2}}} \prod_{i=r+1}^{n} x_{i}^{\lambda-1} .
$$

Therefore, the Bayes reconstructive density $f_{s}^{*}(y \mid \mathbf{x})$, by using equation $(25)$, is as follows

$$
f_{s}^{*}(y \mid \mathbf{x}) \propto \int_{0}^{\infty} \int_{0}^{\infty} f(y \mid \mathbf{x}, \theta, \lambda) g_{1}(\theta \mid \lambda, \mathbf{x}) g_{2}(\lambda \mid \mathbf{x}) h(\theta, \lambda \mid \mathbf{x}) d \theta d \lambda .
$$

It is seen that (28) can not be computed explicitly, therefore, we use a simple sampling procedure to approximate $f_{s}^{*}(y \mid \mathbf{x})$. First, we show that the conditional distribution of $\lambda$ given the data, $g_{2}(\lambda \mid \mathbf{x})$, is log-concave. The $\log$-likelihood of $g_{2}(\lambda \mid \mathbf{x})$ is

$$
\begin{aligned}
\ln g_{2}(\lambda \mid \mathbf{x}) \propto & \left(n-r+a_{1}-1\right) \ln \lambda-b_{1} \lambda-\left(n-r+a_{2}\right) \ln \left(\sum_{i=r+1}^{n} x_{i}^{\lambda}+b_{2}\right) \\
& +(\lambda-1) \sum_{i=r+1}^{n} \ln x_{i} .
\end{aligned}
$$

Now, using Lemma 1 of Kundu (2007), we have $\frac{d^{2}}{d \lambda^{2}} \ln \left(\sum_{i=r+1}^{n} x_{i}^{\lambda}+b_{2}\right) \geqslant$ 0 . Therefore, the conditional density $g_{2}(\lambda \mid \mathbf{x})$ is log-concave. A general method to generate samples from a log-concave density function has been proposed by Devroye (1984). By adopting the method of Devroye (1984), we can generate samples from (27). Now, we use the idea of Geman and Geman (1984) to generate samples from the joint posterior density function (25) using the following algorithm. 
1. Generate $\lambda_{1}$ from $g_{2}(\lambda \mid \mathbf{x})$ in (27) using the method of Devroye (1984).

2. Generate $\theta_{1}$ from $g_{1}\left(\theta \mid \lambda_{1}, \mathbf{x}\right)$.

3. Repeat steps 1 and $2, M$ times and obtain the Monte Carlo (MC) samples $\left(\lambda_{1}, \theta_{1}\right), \ldots,\left(\lambda_{M}, \theta_{M}\right)$.

Note that the steps for generating $\lambda$ from $g_{2}(\lambda \mid \mathbf{x})$ using the Devroye algorithm are as follows:

(i) Compute $c=g_{2}(m \mid \mathbf{x})\left(m\right.$ is the mode of $\left.g_{2}(\lambda \mid \mathbf{x})\right)$.

(ii) Generate $U$ uniform on $[0,2]$, and $V$ uniform on $[0,1]$.

(iii) If $U \leqslant 1$ then $\lambda=U$ and $T=V$, else $\lambda=1-\ln (U-1)$ and $T=$ $V(U-1)$.

(iv) Let $\lambda=m+\frac{\lambda}{c}$.

(v) If $T \leqslant \frac{g_{2}(\lambda \mid \mathbf{x})}{c}$, then $\lambda$ is a sample from $g_{2}(\lambda \mid \mathbf{x})$ else go to Step (i).

Now, using the Monte Carlo (MC) samples $\left(\lambda_{1}, \theta_{1}\right), \ldots,\left(\lambda_{M}, \theta_{M}\right)$, it is possible to obtain the simulation consistent estimator of $f_{s}^{*}(y \mid \mathbf{x})$ as

$$
\widehat{f}_{s}^{*}(y \mid \mathbf{x})=\sum_{i=1}^{M} f\left(y \mid \mathbf{x}, \theta_{i}, \lambda_{i}\right) w_{i}
$$

where

$$
w_{i}=\frac{h\left(\theta_{i}, \lambda_{i} \mid \mathbf{x}\right)}{\sum_{i=1}^{M} h\left(\theta_{i}, \lambda_{i} \mid \mathbf{x}\right)} .
$$

Therefore, the Bayesian reconstructor of $Y$ under the squared error loss can be written as

$$
\begin{aligned}
\widehat{Y}_{B} & =\int_{0}^{x_{r+1}} y \widehat{f}_{s}^{*}(y \mid \mathbf{x}) d y \\
& =\int_{0}^{x_{r+1}} y \sum_{i=1}^{M} f\left(y \mid \mathbf{x}, \theta_{i}, \lambda_{i}\right) w_{i} d y .
\end{aligned}
$$

By using (10) and setting $u=\frac{1-\exp \left(-Y^{\lambda} / \theta\right)}{1-\exp \left(-x_{r+1}^{\lambda} / \theta\right)}$, we get

$$
\widehat{Y}_{B}=\sum_{i=1}^{M}\left[\int_{0}^{1}\left[-\theta_{i} \ln \left[1-u\left(1-\exp \left(-x_{r+1}^{\lambda_{i}} / \theta_{i}\right)\right)\right]\right]^{1 / \lambda_{i}} \frac{u^{s-1}(1-u)^{r-s}}{\operatorname{Beta}(s, r-s+1)} d u\right] w_{i} .
$$




\section{Reconstruction Intervals}

In this section, Bayesian and non-Bayesian approaches are used to obtain reconstruction intervals (RIs) for $Y=X_{s}(1 \leqslant s \leqslant r)$ based on the left type-II censored sample $\mathbf{X}=\left(X_{r+1}, X_{r+2}, \ldots, X_{n}\right)$.

\subsection{Non-Bayesian RIs}

From the pivotal quantity $U$ in (20), it follows that

$$
P\left(b_{1-\alpha / 2}<U<b_{\alpha / 2} \mid x_{r+1}\right)=1-\alpha,
$$

where $b_{\alpha}$ stands for $100 \alpha$ th percentile of $\operatorname{Beta}(s, r-s+1)$. Then by (33), we readily find that

$$
\left(\left[-\theta \ln \left[1-b_{1-(\alpha / 2)}\left(1-\exp \left(-x_{r+1}^{\lambda} / \theta\right)\right)\right]\right]^{1 / \lambda},\left[-\theta \ln \left[1-b_{\alpha / 2}\left(1-\exp \left(-x_{r+1}^{\lambda} / \theta\right)\right)\right]\right]^{1 / \lambda}\right),
$$

is a $100(1-\alpha) \%$ two sided RI for $Y$. When $\lambda$ and $\theta$ are unknown, the parameters in (34) have to be estimated, one can replace $\lambda$ and $\theta$ by their MLEs.

Now let us consider another reconstruction interval for $Y=X_{s}$. By substituting $\lambda$ and $\theta$ in (10) by their MLEs, we can obtain the approximate density of $Y$ given $X_{r+1}=x_{r+1}$ as

$$
\begin{aligned}
\widehat{f}(y \mid \mathbf{x})= & f(y \mid \mathbf{x}, \hat{\lambda}, \hat{\theta}) \\
= & s\left(\begin{array}{l}
r \\
s
\end{array}\right)\left(\frac{\hat{\lambda}}{\hat{\theta}}\right) y^{\hat{\lambda}-1} \exp \left(-\left(y^{\hat{\lambda}} / \hat{\theta}\right)\right)\left[1-\exp \left(-y^{\hat{\lambda}} / \hat{\theta}\right)\right]^{s-1} \\
& \times\left[\exp \left(-y^{\hat{\lambda}} / \hat{\theta}\right)-\exp \left(-x_{r+1}^{\hat{\lambda}} / \hat{\theta}\right)\right]^{r-s}\left[1-\exp \left(-x_{r+1}^{\hat{\lambda}} / \hat{\theta}\right)\right]^{-r} .
\end{aligned}
$$

This conditional density is a unimodal function of $\widehat{U}=\left(\frac{1-\exp \left(-y^{\hat{\lambda}} / \hat{\theta}\right)}{1-\exp \left(-x_{r+1}^{\hat{\lambda}} / \hat{\theta}\right)}\right)$ for $s>1$ and $r>s$ (i.e; $s=2, \ldots, r-1)$. Then, the $100(1-\alpha) \%$ highest conditional density (HCD) RI for $Y$ is

$$
\left(\left[-\hat{\theta} \ln \left[1-w_{1}\left(1-\exp \left(-x_{r+1}^{\hat{\lambda}} / \hat{\theta}\right)\right)\right]\right]^{1 / \lambda},\left[-\hat{\theta} \ln \left[1-w_{2}\left(1-\exp \left(-x_{r+1}^{\hat{\lambda}} / \hat{\theta}\right)\right)\right]\right]^{1 / \lambda}\right),
$$


where $w_{1}$ and $w_{2}$ satisfy the following equations:

$$
1-\alpha=\int_{w_{1}}^{w_{2}} g\left(z \mid x_{r+1}\right) d z
$$

and

$$
g\left(w_{1} \mid x_{r+1}\right)=g\left(w_{2} \mid x_{r+1}\right) .
$$

The equations (36) and (37) can be simplified in terms of incomplete beta function as

$$
B_{w_{2}}(s, r-s+1)-B_{w_{1}}(s, r-s+1)=1-\alpha,
$$

and

$$
\left(\frac{1-w_{2}}{1-w_{1}}\right)^{r-s}=\left(\frac{w_{1}}{w_{2}}\right)^{s-1}
$$

where $B_{t}(a, b)$ is the incomplete beta function. For special case $s=r$, we find a simple expression for $100(1-\alpha) \%$ HCD RI for $Y$, which is

$$
\left(\left[-\hat{\theta} \ln \left[1-\alpha\left(1-\exp \left(-x_{r+1}^{\hat{\lambda}} / \hat{\theta}\right)\right)\right]\right]^{1 / \lambda}, x_{r+1}\right) .
$$

\subsection{Bayesian RIs}

From the Bayes reconstructive density $f_{s}^{*}(y \mid \mathbf{x})$, we can construct a two sided equal-tail reconstruction interval for $Y$. A $100(1-\gamma) \%$ Bayesian reconstruction interval for $Y$ can be obtained by solving the following nonlinear equations simultaneously for the lower bound $L(\mathbf{x})$ and upper bound $U(\mathbf{x})$,

$$
P(Y>L(\mathbf{x}) \mid \mathbf{x})=\int_{L(\mathbf{x})}^{x_{r+1}} f_{s}^{*}(y \mid \mathbf{x}) d y=1-\frac{\alpha}{2}
$$

and

$$
P(Y>U(\mathbf{x}) \mid \mathbf{x})=\int_{U(\mathbf{x})}^{x_{r+1}} f_{s}^{*}(y \mid \mathbf{x}) d y=\frac{\alpha}{2} .
$$

Substituting $f_{s}^{*}(y \mid \mathbf{x})$ by its estimator $\widehat{f}_{s}^{*}(y \mid \mathbf{x})$ in $(29)$, we can obtain $L(\mathbf{x})$ and $U(\mathbf{x})$ from the following equations:

$1-\frac{\alpha}{2}=\sum_{i=1}^{M}\left[\int_{L_{1}}^{1}\left[-\theta_{i} \ln \left[1-u\left(1-\exp \left(-x_{r+1}^{\lambda_{i}} / \theta_{i}\right)\right)\right]\right]^{1 / \lambda_{i}} \frac{u^{s-1}(1-u)^{r-s}}{\operatorname{Beta}(s, r-s+1)} d u\right] w_{i}$, 
and

$$
\frac{\alpha}{2}=\sum_{i=1}^{M} w_{i}\left[\int_{L_{2}}^{1}\left[-\theta_{i} \ln \left[1-u\left(1-\exp \left(-x_{r+1}^{\lambda_{i}} / \theta_{i}\right)\right)\right]\right]^{1 / \lambda_{i}} \frac{u^{s-1}(1-u)^{r-s}}{\operatorname{Beta}(s, r-s+1)} d u\right]
$$

where $L_{1}=\frac{1-\exp \left(-L(\mathbf{x})^{\lambda} / \theta\right)}{1-\exp \left(-x_{r+1}^{\lambda} / \theta\right)}$ and $L_{2}=\frac{1-\exp \left(-U(\mathbf{x})^{\lambda} / \theta\right)}{1-\exp \left(-x_{r+1}^{\lambda} / \theta\right)}$. Numerical methods are generally necessary to solve the above two equations to obtain $L(\mathbf{x})$ and $U(\mathbf{x})$ for a given $\alpha$.

\section{Real Data Analysis}

In order to illustrate the obtained results in the previous sections, the data on the time of breakdown of an insulating fluid in an accelerated test conducted at various test voltages is considered. The data are taken from Nelson (1982) (P. 228, Table. 6.1) and have been used earlier by Viveros and Balakrishnan (1994) and $\mathrm{Wu}$ (2002). In analyzing this data set, Nelson (1982) considered Weibull distribution. The data are

$\begin{array}{lllllll}0.19 & 0.78 & 0.96 & 1.31 & 2.78 & 3.16 & 4.15 \\ 4.67 & 4.85 & 6.50 & 7.35 & 8.01 & 8.27 & 12.06 \\ 31.75 & 32.52 & 33.91 & 36.71 & 72.89 & & \end{array}$

For the sake of comparison between the exact values and the results reconstructed, let us assume that $r=4$ observations are censored on the left so that we only observe the largest $n-r=15$ failure times. Our aim is to reconstruct the four past failure times $\left(X_{1}, X_{2}, X_{3}, X_{4}\right)$. Using different methods discussed in Sections 3 and 4, we obtained Bayesian and non-Bayesian reconstructors for $Y=X_{s}(s \leqslant 4)$. The results are displayed in Table 1. Table 1 also presents the $95 \%$ RI's for $Y=X_{s}(s \leqslant 4)$. For $s=1$ and $s=4$, as mentioned in Section 6.1, the HCD RIs do not exist. Note that for computing Bayesian reconstructors, since we do not have any prior information, we assumed that the priors on $\lambda$ and $\theta$ are improper, i.e. $a_{1}=b_{1}=a_{2}=b_{2}=0$.

By considering the absolute value of the difference between the exact value and the reconstrutors as a criteria, Table 1 indicates that for $s=1,3,4$, $\mathrm{B}$ is the best reconstructor while for $s=3 \mathrm{MLR}$ is the best. Also, all the reconstruction intervals contain the exact observation. It is clear that only one real data analysis does not tell us much more. It should be mentioned here that for computing Bayesian reconstructors, we need to generate the MC samples $\left(\lambda_{1}, \theta_{1}\right), \ldots,\left(\lambda_{M}, \theta_{M}\right)$ using the algorithm described in Subsection 
Table 1. Point and interval reconstructors for the past failure times.

\begin{tabular}{|c|c|c|c|c|c|c|c|c|}
\hline & \multirow[t]{2}{*}{ Excat value } & \multicolumn{4}{|c|}{ Point reconstruction } & \multicolumn{3}{|c|}{ Interval reconstruction } \\
\hline & & $\overline{M L R}$ & BUR & CMR & $\mathrm{B}$ & Pivot meth. & HCD & Bayes meth. \\
\hline$s=1$ & 0.19 & 0.3074 & 0.3445 & 0.2209 & 0.2167 & $(0.0034,1.3316)$ & - & $(0.006,0.8497)$ \\
\hline$s=2$ & 0.78 & 0.7718 & 0.8121 & 0.7196 & 0.5010 & $(0.0722,2.0190)$ & $(0.0412,1.8980)$ & $(0.0537,1.5349)$ \\
\hline$s=3$ & 0.96 & 1.3416 & 1.3775 & 1.3688 & 0.8032 & $(0.2872,2.5029)$ & $(0.3547,2.5989)$ & $(0.1198,2.1278)$ \\
\hline$s=4$ & 1.31 & 2.7800 & 2.0336 & 2.1485 & 1.3924 & $(0.7499,2.7536)$ & - & $(0.3235,2.6784)$ \\
\hline
\end{tabular}

3.2. One can check the convergence of the generated MC samples, using the graphical diagnostics tools like trace plot and plot of autocorrelation function $(\mathrm{ACF})$. Figure 1 shows the trace plot and ACF plot for the parameters $\lambda$ and $\theta$. The trace plot look like a random scatter. Figure 1 also shows the fine mixing of the chains for the parameters. ACF plot shows that chains have very low autocorrelations. In fact, these plots show the rapid convergence of the algorithm.

\section{Numerical Comparisons}

In this section, we intend to compare the performance of the proposed reconstruction methods using Monte Carlo simulations. Comparisons of the reconstructors are performed in terms of biases and mean squared reconstruction errors (MSREs). Different RIs are compared in terms of the average confidence lengths (CL), and coverage percentages (CP). Here we used different $n, r$ and the parameter values $\lambda=1,2$ and $\theta=1$. For computing Bayesian reconstructors, we consider two priors as follows

$$
\begin{array}{lcccc}
\text { Prior I : } & a_{1}=0.0001, & b_{1}=0.0001, & a_{2}=0.0001, & b_{2}=0.0001, \\
\text { Prior II : } & a_{1}=1, & b_{1}=2, & a_{2}=2.5, & b_{2}=1 .
\end{array}
$$

Bayesian point reconstructors are computed from the Gibbs samples under the squared error loss function and with respect to the above priors. For different $n, r, s$ and the above mentioned two priors, Table 2 presents the average biases, and MSREs of the point reconstructors discussed in our paper over 1000 replications. All the computations are performed using the Mathematical software Maple.

From Table 2, the following observations are made: 

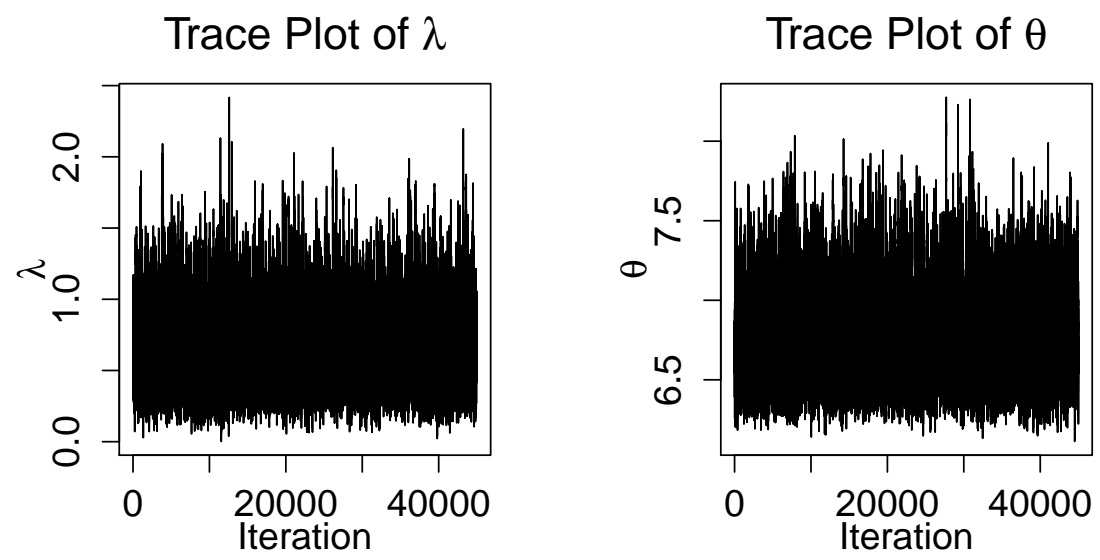

ACF Plot of $\lambda$

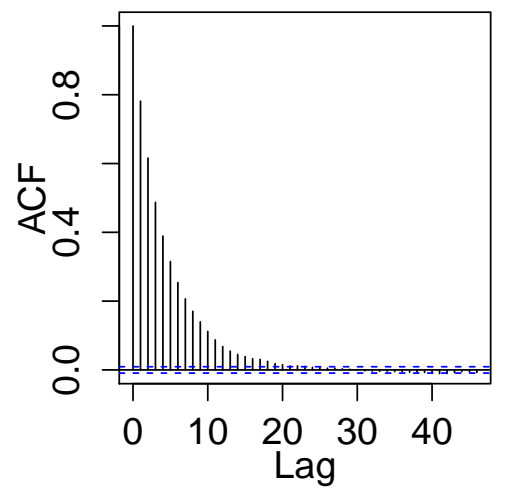

ACF Plot of $\theta$

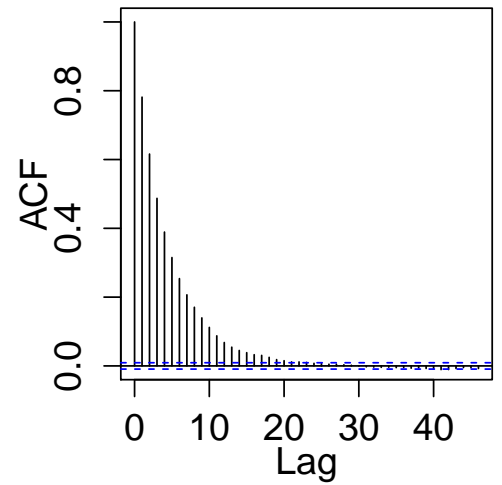

Figure 1. Plots of Markov chains for $\lambda$ and $\theta$.

1. We observe that the CMR is the best point reconstructor among the non-Bayesian point reconstructors. It provides the smallest biases and MSREs in most of the cases considered.

2. It is also seen that the MSREs and biases of the CMR and BUR are very close. The MLR does not work well. 
Table 2. The values of point reconstructions for $\theta=1$ and different $\lambda$.

\begin{tabular}{|c|c|c|c|c|c|c|c|c|c|c|c|c|}
\hline & & & \multicolumn{5}{|c|}{$\lambda=0.75$} & \multicolumn{5}{|c|}{$\lambda=2$} \\
\hline & & & \multirow[t]{2}{*}{ MLR } & \multirow[t]{2}{*}{ BUR } & \multirow[t]{2}{*}{ CMR } & \multicolumn{2}{|c|}{ SER } & \multirow[t]{2}{*}{$\overline{M L R}$} & \multirow[t]{2}{*}{ BUR } & \multirow[t]{2}{*}{ CMR } & \multicolumn{2}{|c|}{ SER } \\
\hline & & & & & & Prior 1 & Prior 2 & & & & Prior 1 & Prior 2 \\
\hline \multirow[t]{2}{*}{$n=5$} & $s=1$ & Bias & 0.057 & 0.175 & 0.152 & 0.052 & 0.041 & 0.162 & 0.155 & 0.132 & 0.113 & 0.101 \\
\hline & & MSRE & 0.101 & 0.088 & 0.079 & 0.069 & 0.063 & 0.109 & 0.091 & 0.084 & 0.075 & 0.069 \\
\hline \multirow[t]{4}{*}{$r=2$} & $s=2$ & Bias & 0.378 & 0.144 & 0.170 & 0.121 & 0.113 & 0.171 & 0.042 & 0.058 & 0.039 & 0.031 \\
\hline & & MSRE & 0.351 & 0.156 & 0.169 & 0.142 & 0.130 & 0.353 & 0.168 & 0.179 & 0.121 & 0.110 \\
\hline & $s=1$ & Bias & 0.011 & 0.076 & 0.056 & 0.009 & 0.007 & 0.042 & 0.091 & 0.059 & 0.053 & 0.048 \\
\hline & & MSRE & 0.033 & 0.035 & 0.033 & 0.029 & 0.019 & 0.051 & 0.044 & 0.039 & 0.034 & 0.027 \\
\hline \multirow[t]{2}{*}{$n=10$} & $s=2$ & Bias & 0.026 & 0.096 & 0.089 & 0.022 & 0.018 & 0.094 & 0.073 & 0.065 & 0.068 & 0.052 \\
\hline & & MSRE & 0.034 & 0.037 & 0.033 & 0.031 & 0.026 & 0.062 & 0.045 & 0.044 & 0.040 & 0.033 \\
\hline \multirow[t]{10}{*}{$r=4$} & $s=3$ & Bias & 0.076 & 0.066 & 0.074 & 0.052 & 0.044 & 0.137 & 0.071 & 0.078 & 0.069 & 0.066 \\
\hline & & MSRE & 0.055 & 0.040 & 0.042 & 0.033 & 0.025 & 0.079 & 0.056 & 0.057 & 0.049 & 0.040 \\
\hline & $s=4$ & Bias & 0.212 & 0.082 & 0.091 & 0.068 & 0.057 & 0.120 & 0.033 & 0.051 & 0.041 & 0.028 \\
\hline & & MSRE & 0.097 & 0.044 & 0.049 & 0.042 & 0.037 & 0.105 & 0.063 & 0.064 & 0.057 & 0.051 \\
\hline & $s=1$ & Bias & 0.021 & 0.012 & 0.003 & 0.003 & 0.002 & 0.011 & 0.062 & 0.032 & 0.032 & 0.026 \\
\hline & & MSRE & 0.003 & 0.002 & 0.002 & 0.002 & 0.001 & 0.018 & 0.019 & 0.016 & 0.013 & 0.011 \\
\hline & $s=2$ & Bias & 0.038 & 0.014 & 0.011 & 0.010 & 0.008 & 0.002 & 0.017 & 0.003 & 0.007 & 0.002 \\
\hline & & MSRE & 0.006 & 0.004 & 0.004 & 0.003 & 0.003 & 0.027 & 0.026 & 0.025 & 0.019 & 0.014 \\
\hline & $s=3$ & Bias & 0.003 & 0.034 & 0.030 & 0.019 & 0.015 & 0.047 & 0.040 & 0.034 & 0.033 & 0.026 \\
\hline & & MSRE & 0.014 & 0.010 & 0.008 & 0.009 & 0.007 & 0.033 & 0.032 & 0.029 & 0.026 & 0.019 \\
\hline \multirow[t]{2}{*}{$n=20$} & $s=4$ & Bias & 0.003 & 0.017 & 0.017 & 0.034 & 0.029 & 0.056 & 0.036 & 0.036 & 0.029 & 0.024 \\
\hline & & MSRE & 0.017 & 0.016 & 0.010 & 0.010 & 0.009 & 0.043 & 0.040 & 0.038 & 0.034 & 0.027 \\
\hline \multirow[t]{6}{*}{$r=7$} & $s=5$ & Bias & 0.031 & 0.024 & 0.029 & 0.047 & 0.035 & 0.031 & 0.025 & 0.029 & 0.025 & 0.020 \\
\hline & & MSRE & 0.020 & 0.018 & 0.013 & 0.012 & 0.010 & 0.050 & 0.044 & 0.039 & 0.037 & 0.032 \\
\hline & $s=6$ & Bias & 0.054 & 0.018 & 0.029 & 0.055 & 0.043 & 0.054 & 0.018 & 0.028 & 0.021 & 0.013 \\
\hline & & MSRE & 0.028 & 0.026 & 0.034 & 0.022 & 0.016 & 0.062 & 0.058 & 0.043 & 0.040 & 0.037 \\
\hline & $s=7$ & Bias & 0.090 & 0.018 & 0.034 & 0.069 & 0.060 & 0.090 & 0.018 & 0.034 & 0.023 & 0.016 \\
\hline & & MSRE & 0.030 & 0.025 & 0.021 & 0.019 & 0.017 & 0.079 & 0.069 & 0.051 & 0.046 & 0.041 \\
\hline
\end{tabular}


Table 3. Average confidence/credible length and coverage percentage for $\theta=1$ and different $\lambda$.

\begin{tabular}{|c|c|c|c|c|c|c|c|c|c|c|}
\hline & & & \multicolumn{4}{|c|}{$\lambda=0.75$} & \multicolumn{4}{|c|}{$\lambda=2$} \\
\hline & & & \multirow[t]{2}{*}{ Pivot. Meth. } & \multirow[t]{2}{*}{ HCD } & \multicolumn{2}{|c|}{ Bay. Meth. } & \multirow[t]{2}{*}{ Pivot. Meth. } & \multirow[t]{2}{*}{$\mathrm{HCD}$} & \multicolumn{2}{|c|}{ Bay. Meth. } \\
\hline & & & & & Prior 1 & Prior 2 & & & Prior 1 & Prior 2 \\
\hline \multirow[t]{2}{*}{$n=5$} & $s=1$ & $\mathrm{CL}$ & 0.527 & - & 0.461 & 0.444 & 0.552 & - & 0.501 & 0.488 \\
\hline & & $\mathrm{CP}$ & 0.875 & - & 0.892 & 0.903 & 0.905 & - & 0.914 & 0.920 \\
\hline \multirow[t]{4}{*}{$r=2$} & $s=2$ & $\mathrm{CL}$ & 0.540 & - & 0.502 & 0.485 & 0.589 & - & 0.562 & 0.553 \\
\hline & & $\mathrm{CP}$ & 0.918 & - & 0.925 & 0.929 & 0.923 & - & 0.929 & 0.933 \\
\hline & $s=1$ & $\mathrm{CL}$ & 0.319 & - & 0.281 & 0.263 & 0.492 & - & 0.474 & 0.463 \\
\hline & & $\mathrm{CP}$ & 0.842 & - & 0.862 & 0.877 & 0.892 & - & 0.911 & 0.918 \\
\hline \multirow[t]{2}{*}{$n=10$} & $s=2$ & $\mathrm{CL}$ & 0.390 & 0.282 & 0.348 & 0.327 & 0.549 & 0.471 & 0.527 & 0.503 \\
\hline & & $\mathrm{CP}$ & 0.861 & 0.849 & 0.894 & 0.906 & 0.907 & 0.885 & 0.918 & 0.926 \\
\hline \multirow[t]{10}{*}{$r=4$} & $s=3$ & $\mathrm{CL}$ & 0.432 & 0.406 & 0.425 & 0.413 & 0.562 & 0.504 & 0.548 & 0.530 \\
\hline & & $\mathrm{CP}$ & 0.910 & 0.893 & 0.919 & 0.924 & 0.918 & 0.902 & 0.927 & 0.935 \\
\hline & $s=4$ & CL & 0.493 & - & 0.451 & 0.440 & 0.628 & - & 0.614 & 0.601 \\
\hline & & $\mathrm{CP}$ & 0.918 & - & 0.924 & 0.930 & 0.928 & - & 0.935 & 0.942 \\
\hline & $s=1$ & CL & 0.198 & - & 0.166 & 0.148 & 0.486 & - & 0.465 & 0.448 \\
\hline & & $\mathrm{CP}$ & 0.904 & - & 0.911 & 0.921 & 0.902 & - & 0.917 & 0.924 \\
\hline & $s=2$ & $\mathrm{CL}$ & 0.209 & 0.158 & 0.186 & 0.173 & 0.507 & 0.471 & 0.493 & 0.484 \\
\hline & & $\mathrm{CP}$ & 0.921 & 0.905 & 0.925 & 0.932 & 0.911 & 0.891 & 0.920 & 0.929 \\
\hline & $s=3$ & $\mathrm{CL}$ & 0.222 & 0.186 & 0.213 & 0.202 & 0.538 & 0.497 & 0.526 & 0.515 \\
\hline & & $\mathrm{CP}$ & 0.936 & 0.917 & 0.937 & 0.939 & 0.916 & 0.905 & 0.926 & 0.930 \\
\hline \multirow[t]{2}{*}{$n=20$} & $s=4$ & $\mathrm{CL}$ & 0.250 & 0.210 & 0.238 & 0.227 & 0.604 & 0.544 & 0.590 & 0.578 \\
\hline & & $\mathrm{CP}$ & 0.938 & 0.924 & 0.931 & 0.928 & 0.925 & 0.919 & 0.935 & 0.941 \\
\hline \multirow[t]{6}{*}{$r=7$} & $s=5$ & $\mathrm{CL}$ & 0.265 & 0.227 & 0.253 & 0.245 & 0.655 & 0.607 & 0.643 & 0.632 \\
\hline & & $\mathrm{CP}$ & 0.940 & 0.927 & 0.940 & 0.942 & 0.938 & 0.924 & 0.940 & 0.943 \\
\hline & $s=6$ & CL & 0.290 & 0.238 & 0.274 & 0.258 & 0.680 & 0.638 & 0.661 & 0.648 \\
\hline & & $\mathrm{CP}$ & 0.946 & 0.939 & 0.947 & 0.952 & 0.946 & 0.940 & 0.950 & 0.951 \\
\hline & $s=7$ & $\mathrm{CL}$ & 0.319 & - & 0.296 & 0.282 & 0.709 & - & 0.685 & 0.672 \\
\hline & & $\mathrm{CP}$ & 0.955 & - & 0.956 & 0.956 & 0.947 & - & 0.954 & 0.955 \\
\hline
\end{tabular}


3. Comparing the two Bayesian reconstructors based on two priors I and II, as anticipated, Bayesian reconstructors based on informative prior II perform better than Bayesian reconstructors based on improper prior I in terms of both biases and MSPEs.

4. We also observe that Bayesian reconstructors based on informative prior II are better than all the non-Bayesian reconstructors.

5. From Table 2, it is also observed that for fixed $r$ and $n$, as $s$ increases the MSREs decrease as expected.

We also computed $95 \%$ RIs for $Y=X_{s}(s \leqslant r)$ by using the results given in Section 4. In Table 3, the means and coverage probabilities of the lengths of $95 \%$ the RIs are reported. As shown in Table 3:

1. The HCD RIs are the shortest RIs.

2. It is also observed that the RIs based on informative prior II perform better than the RIs based on improper prior I, and the RIs provided by pivotal method, in terms of both average lengths and coverage probabilities.

3. It is evident that the RIs based on the informative prior II provide the highest coverage probabilities.

\section{Acknowledgement}

The authors are grateful to the two anonymous referees for making many helpful comments and suggestions on an earlier version of this paper.

\section{References}

Aitchison, J. and Dunsmore, I.R. (1975). Statistical Prediction Analysis, Cambridge University Press.

Asgharzadeh, A., Ahmadi, J., Mirzazadeh Ganji, Z. and Valiollahi, R. (2012). Reconstruction of the Past Failure Times for the Proportional Reversed Hazard Rate Model. Journal of Statistical Computation and Simulation, 82, 475-489.

Asgharzadeh, A., Mohammadpour, M. and Ganji, Z.M. (2014). Estimation and Reconstruction Based on Left Censored Data from Pareto Model, Journal of the Iranian Statistical Society, 13, 151-175.

J. Statist. Res. Iran 14 (2017): 31-51 
Bagger, J. (2005). Wage Growth and Turnover in Denmark, University of Aarhus, Denmark.

Bhaumik, D.L., Santra, S., Aryal, S., and Gibbons, R. (2009). One-sided Simultaneous Prediction Limits for Left-censored Normal Random Variables. Sankhya, 70-B, 248-266.

Balakrishnan, N. (1989). Approximate MLE of the Scale Parameter of the Rayleigh Distribution with Censoring. IEEE Transactions on Reliability, 38, 355-357.

Balakrishnan, N. and Varadan, J. (1991). Approximate MLEs for the Location and Scale Parameters of the Extreme Value Distribution with Censoring. IEEE Transactions on Reliability, 40, 146-151.

Balakrishnan, N., Doostparast, M. and Ahmadi, J. (2009). Reconstruction of Past Records, Metrika, 70, 89-109.

Devroye, L. (1984). A Simple Algorithm for Generating Random Variates with a Log-concave Density. Computing, 33, 247-257.

El-Adll, M.E. and Aly, A.E. (2016). Reconstructing Past Fractional Record Values. Journal of the Egyptian Mathematical Society, 24, 622-628.

Geman, S. and Geman, A. (1984). Stochastic Relaxation, Gibbs Distributions and the Bayesian Restoration of Images. IEEE Transactions on Pattern Analysis and Machine Intelligence, 6, 721-740.

Johnson, N., Kotz, S. and Balakrishnan, N. (1994). Continuous Univariate Distributions, Vol 2. John Wiley and Sons, New York.

Kaminsky, K.S. and Rhodin, L.S. (1985). Maximum Likelihood Prediction. Annals of the Institute of Statistical Mathematics, 37, 507-517.

Khatib, B., Ahmadi, J. and Razmkhah, M. (2013). Bayesian Reconstruction of the Missing Failure Times in Exponential Distribution. Journal of Statistical Computation and Simulation, 83, 501-517.

Klimczak, M. and Rychlik, T. (2005). Reconstruction of Previous Failure Times and Records, Metrika, 61, 277-290.

Kundu, D. (2007). On Hybrid Censoring Weibull Distribution. Journal of Statistical Planning and Inference, 137, 2127-2142.

Kundu, D. (2008). Bayesian Inference and Life Testing Plan for the Weibull Distribution in Presence of Progressive Censoring. Technometrics, 50, 144-154.

Mitra, S. and Kundu, D. (2008). Analysis of Left Censored Data from the Generalized Exponential Distribution. Journal of Statistical Computation and Simulation, 78, 669-679.

Nelson, W. (1982). Applied Life Data Analysis, John Wiley and Sons, New York. 
Noor, F. and Aslam, M. (2013). Bayesian Inference of the Inverse Weibull Mixture Distribution Using Type-I Censoring. Journal of Applied Statistics, 40, 1076-1089.

Raqab, M.Z., Asgharzadeh, A. and Valiollahi, R. (2010). Prediction for Pareto Distribution based on Progressively Type-II Censored Samples. Computational Statistics and Data Analysis, 54, 1732-1743.

Raqab, M.Z. and Nagaraja, H.N. (1995). On Some Predictors of Future Order Statistics. Metron, 53, 185-204.

Razmkhah, M., Khatib, B. and Ahmadi, J. (2010). Reconstruction of Order Statistics in Exponential Distribution, Journal of the Iranian Statistical Society, 9, 21-40.

Scallan, A.J. (1999). Regression Modelling of Interval-censored Failure Time Data Using the Weibull Distribution. Journal of Applied Statistics, 26, 613-618.

Viveros, R. and Balakrishnan, N. (1994). Interval Estimation of Life Characteristics from Progressively Censored Data. Technometrics, 36, 84-91.

Wu, S.J. (2002). Estimation of the Parameters of the Weibull Distribution with Progressive Censored Data, Journal of the Japan Statistical Society, 32, 155-163.

\section{A. Asgharzadeh}

Department of Statistics,

University of Mazandaran,

Babolsar, Iran.

email: a.asgharzadeh@umz.ac.ir

\section{R. Valiollahi}

Department of Mathematics, Statistics and Computer Science,

Semnan University,

Semnan, Iran.

email:valiollahi.reza@gmail.com

\section{Z. Mirzazadeh}

Department of Statistics, University of Mazandaran, Babolsar, Iran. email: zmganji@gmail.com

\section{J. Ahmadi}

Department of Statistics, Ferdowsi University of Mashhad, Mashhad, Iran.

email: ahmadi-j@um.ac.ir 
